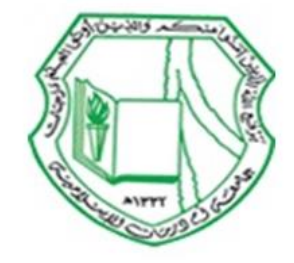

\title{
اثر استخدام الرمل السيليكي على مقاومة الخرسانة
}

\section{Fathelrahman M. Adam ${ }^{1, *}$, Omer E. A. ${ }^{2}$, Mohammed I. Ali ${ }^{2}$, and Salih E. M. ${ }^{3}$}

1 Civil Engineering Department, Nile Valley University, Atbara, Sudan

2 Civil Engineering Department, Kordofan University, El-Obied, Sudan.

3 Civil Engineering Department, Sudan University of Science and Technology, Khartoum, Sudan

* Corresponding author: Fathelrahman M. Adam (e-mail: $\underline{\text { fat470@yahoo.com). }}$ ).

Article history: Received 25 April 2020, Received in revised form 4 November 2020, Accepted 11 November 2020

الملخص: تم في هذا البحث عمل دراسة باستخدام الرمل السيليكي بنقاوة 95\% ومعرفة مدى تأثيره على مقاومة الخرسانة عند استخدامه بديلاً عن الرمل

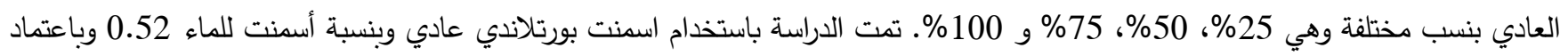
المواصفات البريطانية في تصميم الخلطة. أستخدمت النسبة 1:5:3 1:5 للنسب المختلفة للمواد المكونة للخلطة. تم استخدام خلطة مرجعية خالية من الرمل السيليكي بما يعني نسبة 0\% من الرمل السيليكي. أجريت اختبارات مقاومة الخرسانة للمكعبات بعمر 7 يوم و 28 يوم وأظهرت النتائج المتحصل عليها

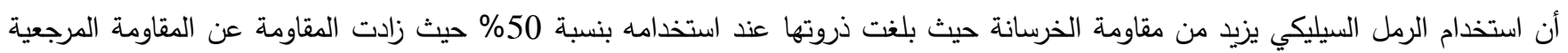

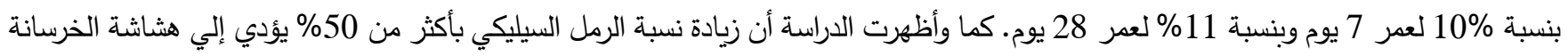

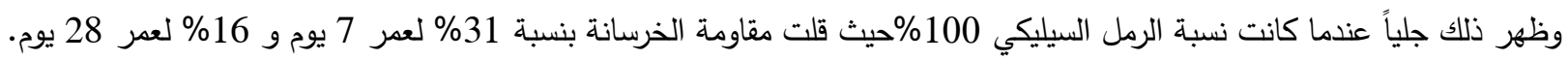

$$
\text { الكلمات المفتاحية: الرمل السيليكي، تصميم الخلطة، خواص الخرسانة، مقاومة الخرسانة. }
$$

ABSTRACT In this research, a study of using silica sand of a purity reaches $95 \%$ was done in order to know its effects on the strength of concrete when it used as an alternative to the normal sand by a different ratio of $25 \%, 50 \%, 75 \%$ and $100 \%$. The study was done using Normal Portland Cement with a water/cement ratio of 0.52 and the mix design was done according to the British Standard Method. The ratio 1:1.5:3 was used for the different materials composed of the mix design. A reference mix design was used without containing any silica sand by means of $0 \%$ ratio of silica sand. Compression tests for the strength of concrete have been done for cubes of 7 days and 28 days age of curing and the results obtained appeared that the using of silica sand increases the concrete strength with reaching the peak at a ratio of $50 \%$ of silica sand where the strength was increased for the 7 days and 28 days by a ratio of $10 \%$ and $11 \%$ more than the strength of reference mix design respectively. Also, the study appears that the increase of silica sand by more than $50 \%$, leads to the brittleness of concrete and this has been seen clearly when the ratio of silica sand reached $100 \%$ since the strength of concrete be down by $31 \%$ for 7 days and by $16 \%$ for the 28 days.

Keywords: Silica Sand, Mix Design, Properties of Concrete, Strength of Concrete.

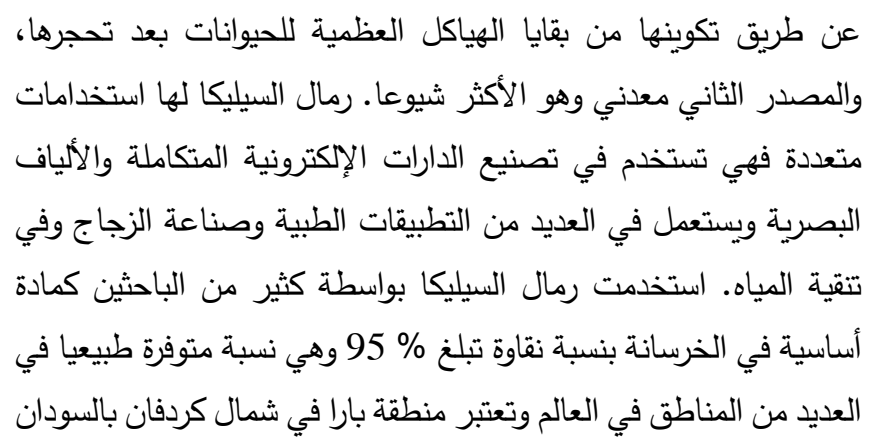

\section{1}

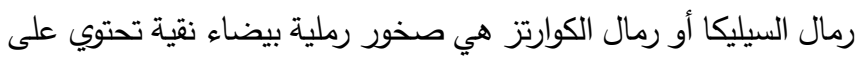

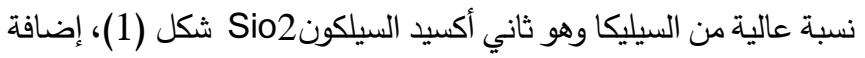

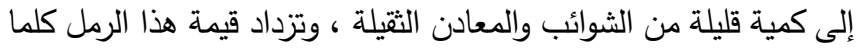
انخفضت نسبة الثوائب التي يحتوي عليها. يوجد مصدران للسيلكا، الأول عضوي مثله مثل النفط والفوسفات تكون على مدى ملايين السنين، وذلك 
إجراء التجارب المعملية يتم اختيار المواد وفق الخواص المطلوبة للخرسانة وتوجد تقاصيل وافية بالمرجع [4].

\section{3. - واص الخرسان}

تمتاز الخرسانة بمقاومتها العالية للضغط باعتبار أنها من المواد القاصفة

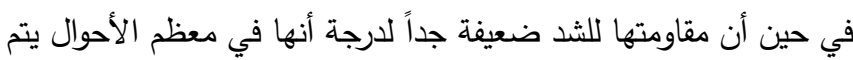
تجاهل هذه المقاومة. ولمقابلة ضعف الخرسانة في الثد يتم استخدام حديد

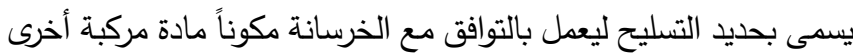

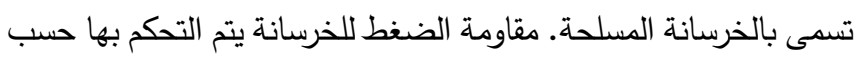

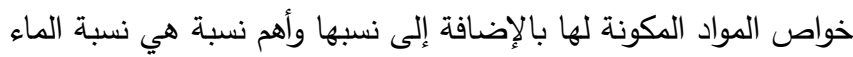
للأسمنت وهذه النسبة لها أهمية كبيرة في علو وانخفاض مقاومة الخرسانة بالإضافة للخواص الأخرى مثل الانكماش، ومقاومة التآكل والكثافة. يتم

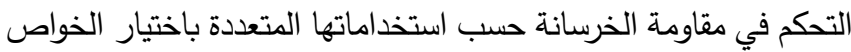
والنسب المحدة للمواد. يتم تحديد أقصى قوة تحمل للضغط في الخرسانة بعمل اختبار الضغط باستخدام ماكينة الكسر وذلك بعمل عينات قياسية منها المكعب كما في المواصفة البريطانية والأوربية أو الاسطوانية كما في

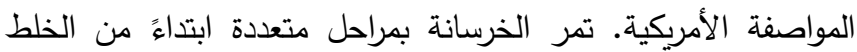

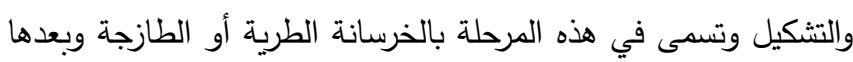
تتتقل لمرحلة التصلد وتصاحب هذه المرحلة عملية مهمة تسمى بالمعالجة وهي القيام بعمل الترطيب اللازم للخرسانة بحيث يتم الحفاظ على نسبة الماء للأسمنت داخل الخرسانة بغرض الحصول على المقاومة المطلوبة بصورة مضطردة مع الزمن ولقد حددت معظم الدراسات أن مقاومة

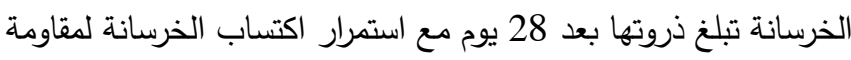
إضافية مع تقدم عمرها لكن بصورة أقل وأبطأ.

\section{4.تصميم الخلطة الخرسانية}

يقصد بتصميم الخلطة الخرسانية هو تحديد النسب المكونة لها بما يتقق مع المتطلبات المطلوبة وفق الاستخدامات المتعددة للخرسانة. ويتم ذلك بطرق حسابية تضع في الاعتبار خواص المواد المستخدمة والخواص المطلوبة في الخرسانة المتصلدة (مثل مدى المقاومة للأحمال أو المقاومة للبري

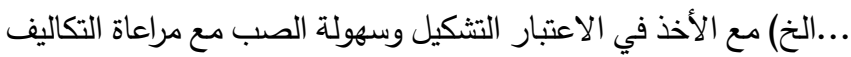

$$
\text { الاقتصادية حسب نوع العمل الإنشائي المطلوب. }
$$

توجد طرق متعددة لتصميم الخلطة منها الطريقة البريطانية حسب الابت المواصف 1883-11683 1881 ويتم فيها تصميم الخلطات الخرسانية من خلال خمسة مراحل أساسية يتم فيها تحديد العوامل المهمة

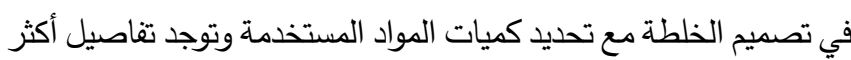
بالمرجع [5]. طريقة أخرى هي الطريقة الأمريكية حسب المواصفة ACI

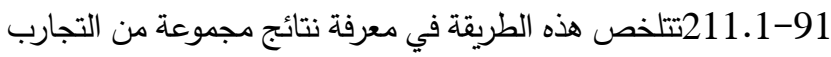

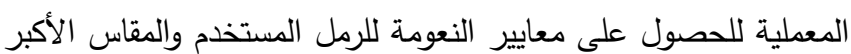

غنية بهذه الرمال وتتواجد تحت سطح الأرض بعمق يتراوح من متر إلى متر ونصف.. توجد دراسات متعددة لإستخدام الرمل السيليكي في الخرسانة حيث توصلت نهلة ناجي هلال وآخرون [1] إلى أن استبدال الرمل السيليكي بالرمل العادي بنسبة 75\% يحسن من مقاومة الخرسانة بنسبة 42.9\% كما

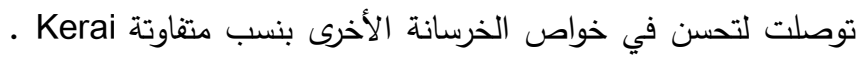
[2 [2 [2 توصلا إلى تحسن في مقاومة الخرسانة بنسبة 1.2\% عند استخدام الرمل السيليكي بنسبة 3]\%.Sravani Pachipala 50 [3صل على زيادة في مقاومة

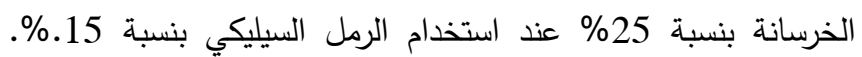
يتضمن البحث أيضاً نتائج مأخوذة من مراجع أخري تم استخدام الرمل السيليكي فيها بنسب متفاوتة أدت إلي نتائج متباينة في قيم مقاومة الخرسنة

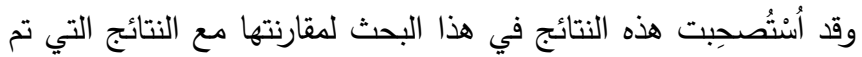

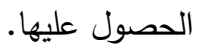
يهدف هذا البحث إلي استخدام الرمل السيليكي بنسب مختلفة كبديل للرمل العادي في الخلطة الخرسانية وذلك لمعرفة أثر هذه النسب على لئى مقاومة

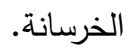

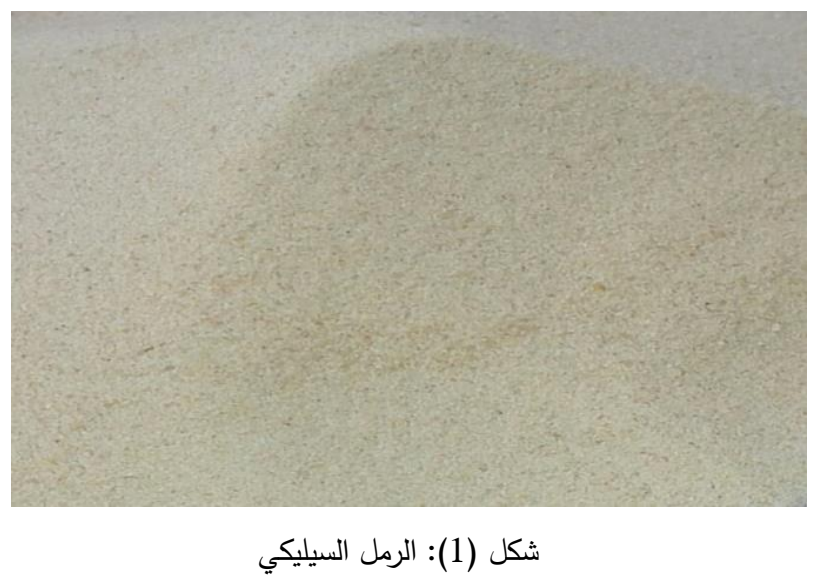

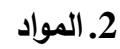

الخرسانة هي مادة مركبة تتكون من عدة مواد منها الركام الخشن

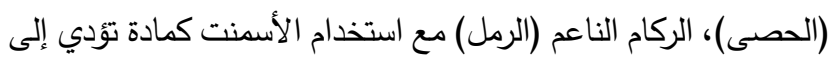

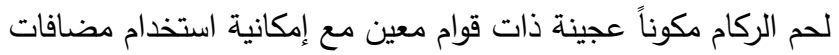
لإضافة بعض الخواص ويستخدم الماء للخلطويضاف بمقدار معين وهو لازم لعملية تفاعل الأسمنت للحصول على اللحم والربط اللازم بين

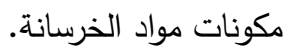
المواد المكونة للخرسانة لها خواص عديدة منها الكيميائي، الفيزيائي والميكانيكي ويتم تحديد هذه الخواص عن طريق التجارب المعملية. بعد 
جدول (2): الفرق المئوي بين مقاومة الضغط للنسبة المختلفة من الرمل السيليكي مع المقاومة المرجعية - معاية

الفرق المئوي

\begin{tabular}{|c|c|c|}
\hline & \multirow{2}{*}{ الخلطة } \\
\hline عمر 28 يوم & عمر 7 يوم & \\
\hline $2 \%$ & $-2 \%$ & 25\% رمل سيليكي \\
\hline $11 \%$ & $10 \%$ & 50\% رمل سيليكي \\
\hline$-7 \%$ & $-7 \%$ & 75\% رمل سيليكي \\
\hline$-16 \%$ & $-31 \%$ & 100\% رمل سيليكي \\
\hline
\end{tabular}

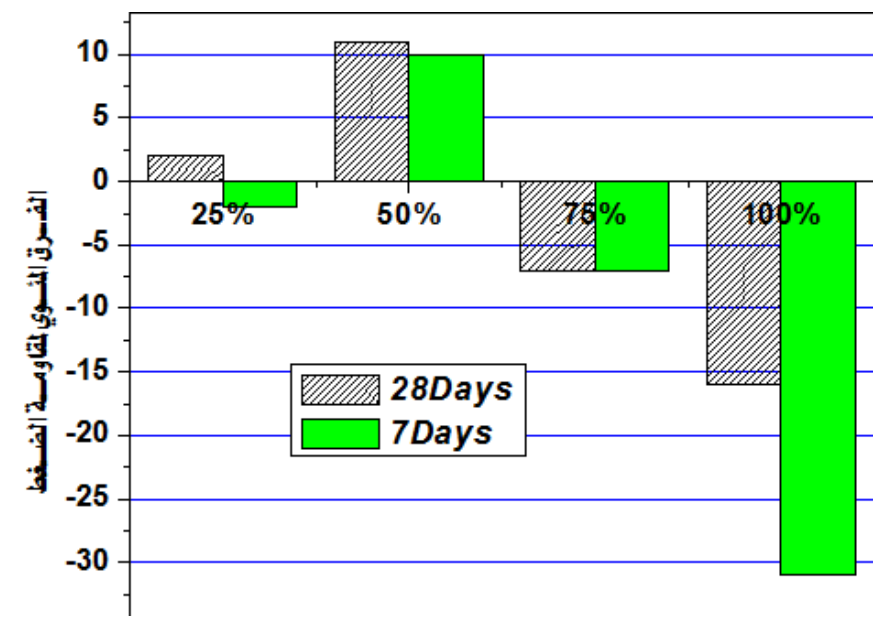

شكل (3): الفرق المئوي لمقاومة ضغط الخرسانة للنسب المختلفة من الرمل السيليكي مقارنة مع الخلطة المرجعية

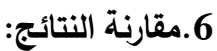

فيما يلي بالثكل (4) مقارنة للنتائج المتحصل عليها لمقاومة الخرسانة

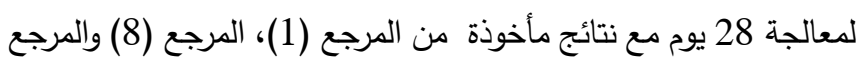

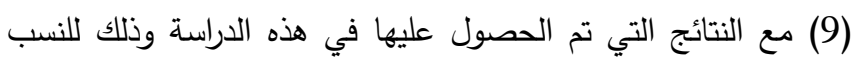

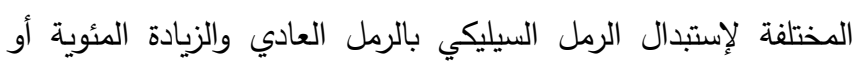
النقصان عن الدقاومة المرجعية للخلطة التي يستخدم فيها الرمل العادي

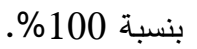

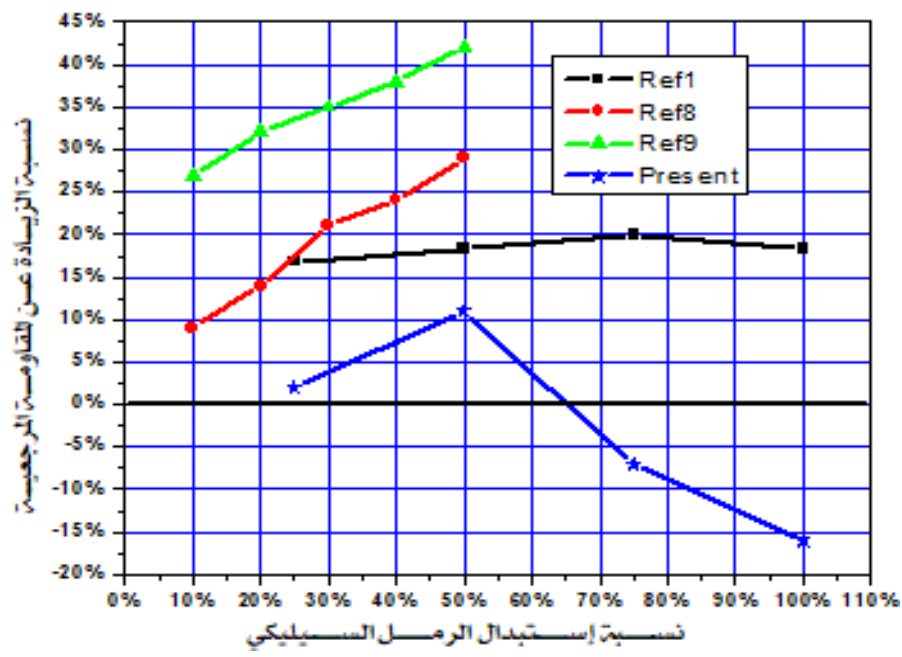

شكل (4): مقارنة نسبة الزيادة في مقاومة الخرسانة في 28 يوم عن المقاومة

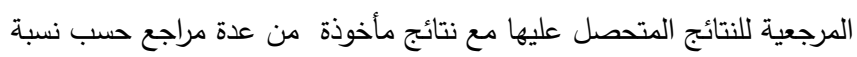
الرمل السيليكي بالخلطة
للركام الكبير ووحدة الحجم للركام الكبير الجاف الدكوك والوزن النوعي

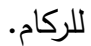

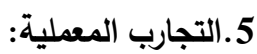

تم عمل تصميم للخلطة وفق منطلبات المدونة البريطانية 18:1983-11681حسب المواد المكونة للخلطة بنسبة 1:1/21:3. الأسمنت المستخدم هو أسمنت بورتلاندي عادي والمياه هي مياه نظيفة صالحة للشرب بنسبة من الأسمنت بمقدار 0.52. تم عمل عدة خلطات بإضافة الرمل السيليكي واستبداله بالرمل العادي

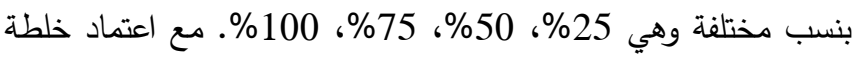
مرجعية بدون أي إضافة للرمل السيليكي. تم إعداد الخلطات المتعددة بالطريقة المتبعة من خلط للمواد بالنسب حسب لمبل تصميم الخلطة ووضعها في القوالب القياسية 150x150x150 والدمك بالطريقة الموصوفة بالمواصفات ومن ثم المعالجة. اختبار مقاومة الضغط للخرسانة تم عمله للمكعبات القياسية للخلطات

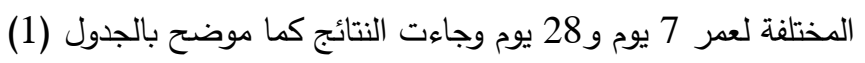
والثكل (2). جدول (1): مقاومة ضغط الخرسانة للنسب المختلفة من الرمل السيليكي مقاومة الضغط للخرسانة

\begin{tabular}{|c|c|c|}
\hline عمر 28 يوم & عمر 7 يوم & \\
\hline 26.57 & 26.03 & لخلطة المرجعية (0\%) \\
\hline 27.00 & 25.63 & 25\% رمل سيليكي \\
\hline 29.37 & 28.53 & 50\% رمل سيليكي \\
\hline 24.73 & 24.3 & 75\% رمل سيليكي \\
\hline 22.4 & 18 & 100\% رمل سيليكي \\
\hline
\end{tabular}

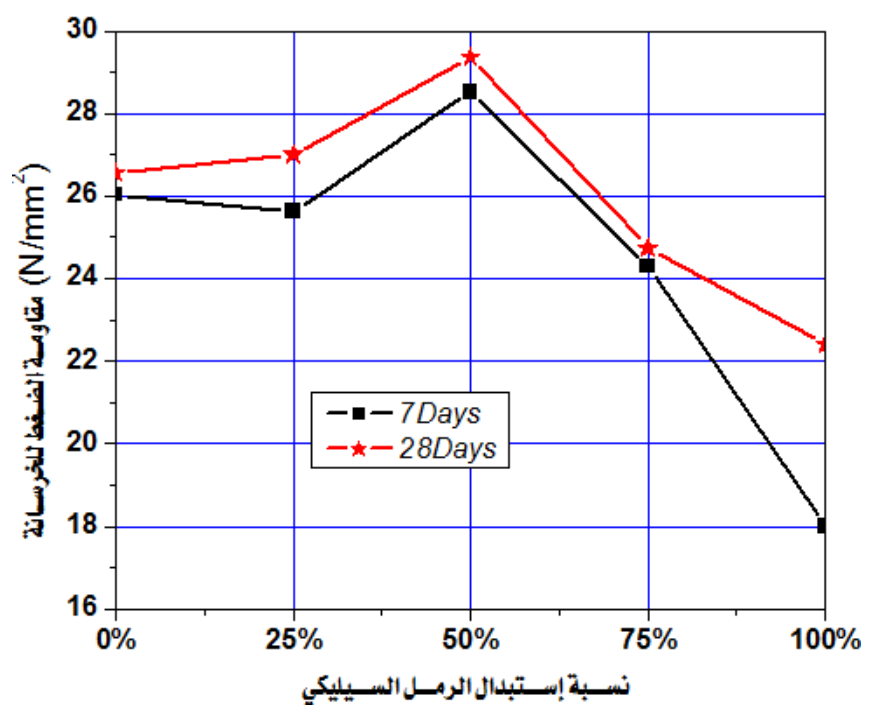

شكل (2): مقاومة ضغط الخرسانة للنسب المختلفة من الرمل السيليكي 
• من الضروري معرفة الخواص الأخرى للخرسانة المصاحبة لاستخدام الرمل السيليكي بنسبه المختلفة.
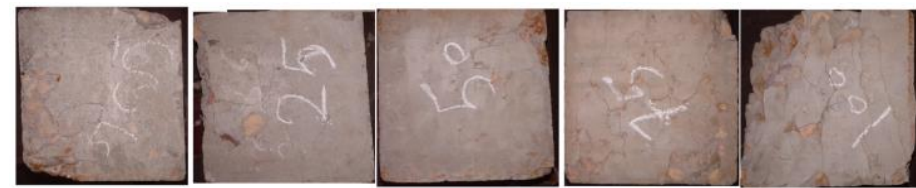

شكل (5): شكل التهشيم لمكعب الخرسانة للنسب المختلفة من الرمل السيليكي

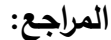

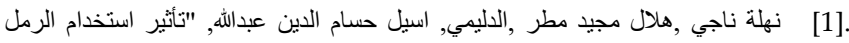

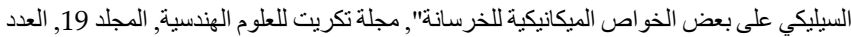

.2012, 44-22, 4

[2]. Kerai Jignesh, Shraddha R. Vaniya, "Effect of use of Silica Sand as Fine Material in Concrete", International Journal for Innovative Research in Science \& Technology IJIRST, Volume 2, Issue 01, p266-271, 2015.

[3]. Sravani Pachipala, "A Study on Mechanical Properties of Concrete Using Silica Sand as Partial Replacement of Cement", International Journal of Civil Engineering, Volume 4 Issue 5, p34-39, May 2017.

[4]. M. Neville, "Properties of Concrete", Pitman publishing limited, London, 3rd edn, 1981.

[5]. Kong F.K., Evans R.H., "Reinforced and Prestressed Concrete”, 3rd Edition, CRC Press, 2014.

[6]. ACI Standard 211.1-91,"Standard Practice for Selecting Proportions for Normal, Heavyweight and Mass Concrete", American Concrete Institute - Farmington Hills, Michigan, 1991.

[7]. BS 1881-116:1983, "Method of Determination of Compressive Strength of Concrete Cube", British Standard Institution.

[8]. Sawan B., Girirajsinh G., Uttam C., Harshil K., "A Parametric Study on Partial Replacement of Normal Sand by Silica Sand in Concrete", Project Report, Atmiya Institute Of Technology And Science Rajkot, Gujarat Technological University, Ahmedabad, 2018. [9]. Hanifi Binici, Orhan Aksogan, "Durability of concrete made with natural granular granite, silica sand and powders of waste marble and basalt as fine aggregate", Journal of Building Engineering, Pages 109-121,

https://doi.org/10.1016/j.jobe.2018.04.022, 2018.

Fathelrahman Mohamed Adam Associate professor, Department of Civil Engineering, Nile Valley University, I studied civil engineering at Sudan University of Science \& Technology and received my postgraduate studies at the same University in the field of finite element method as application in shell analysis.

My Work in Nile Valley University is continued for 23 years, 5 years of that I join work at Jazan University (KSA).

My experience in teaching is concentrated in subjects, structural analysis especially numerical methods like flexibility, stiffness and finite element methods also reinforced concrete design, strength of materials, computer application and foundation engineering.

I standed in the head of exam committee (Control), head of civil engineering department and head of engineering management and now holding a dean of academic affairs.

I have many published papers and two books published in lap lambert academic publishing.
7.مناقشة النتائج:

من خلال النتائج المتحصل عليها لمقاومة الضغط لكل خلطة ونتائج

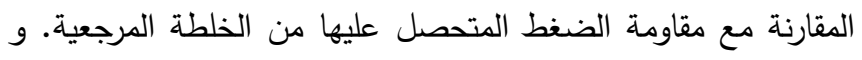

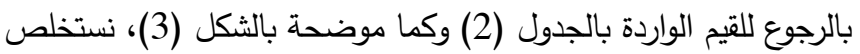
النقاط التالية:

• مقاومة الضغط المتحصل عليها في عمر 7 يوم قلت في كل النسب

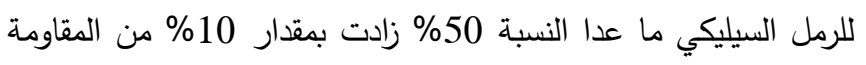
15

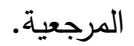
• مقاومة الضغط المتحصل عليها في عمر 28 يوم زادت بنسبة بسيطة للنسبة 25\% بينما زادت بنسبة

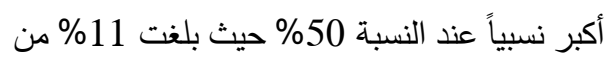
قيمة مقاومة الضغط للخلطة المرجعية. • بزيادة الرمل السيليكي بأكثر من 50\% تلاحظ تراجع قيمة المقاومة بصورة مضطردة بلغت ذروتها عند الاستبدال الكامل للرمل العادي بالرمل

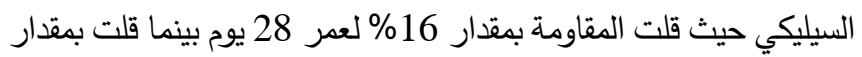

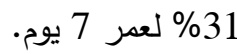

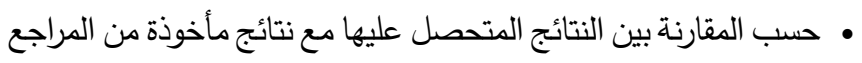
الأخري كما يبين الثكل (4)، نجد أن معظمها يتفق على أن الزيادة

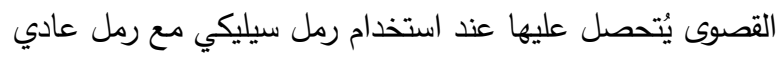

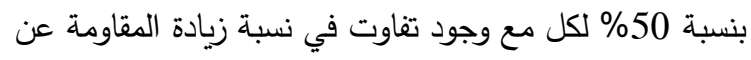

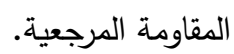

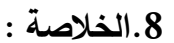

$$
\text { نخلص من هذه الدراسة إلى الآتي: }
$$

• أن استخدام الرمل السيليكي كبديل للرمل يزيد من مقاومة الخرسانة التاني

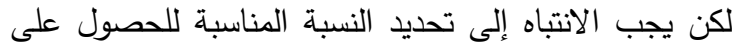

الزيادة ذات الأفضلية وفي هذه الدراسة وجد أن النسبة المناسبة

لاستخدامه كبديل للرمل بنسبة 50.

• في حالة استبدال الرمل بأكثر من 50\% تزداد هشاشة الخرسانة

باطراد وذلك واضح من شكل التهشيم كما في الشكل (5)

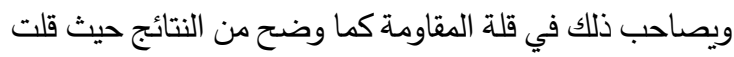
المقاومة بمقدار يقترب من الثلث عندما كانت نسبة استبدال

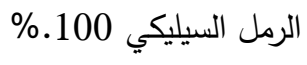

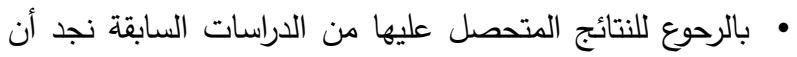

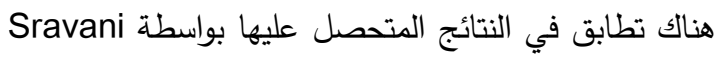

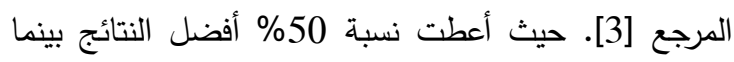
تباينت المراجع الأخرى في هذه النسبة بين أكثر منها وأقل منها

$$
\text { مما يؤكد ضرورة تحديد هذه النسبة. }
$$

